\title{
Xanthogranulomatous endometritis: a report of two Korean cases with cytologic findings
}

\author{
Ji Min $\mathrm{Na}^{1}$, Min Hye Kim ${ }^{1}$, Gyung Hyuck Ko ${ }^{1,2,3}$, Jeong Kyu Shin ${ }^{2,3,4}$ \\ 'Department of Pathology, Gyeongsang National University Hospital, Jinju; ${ }^{2}$ Gyeongsang National University College of Medicine, Jinju; \\ ${ }^{3}$ Gyeongsang Institute of Health Science, Jinju; ${ }^{4}$ Department of Obstetrics and Gynecology, Gyeongsang National University Hospital, Jinju, Korea
}

Xanthogranulomatous inflammation is a form of chronic inflammation characterized by aggregation of lipid-laden foamy histiocytes that occurs most commonly in the kidney and gallbladder. Xanthogranulomatous endometritis (XGE) is rareless than 30 cases have been reported worldwide [1-10], and none were in Korea. We report here two cases of XGE in Korea along with its cytologic findings, which have not been described previously.

\section{CASE REPORT}

\section{Case 1}

A 78-year-old woman presented with a chief complaint of yellow pus-like vaginal discharge for 15 days. She had a history of hypertension for 10 years and was on medication for heart failure. On ultrasonography, her uterus was found to be enlarged, and fluid collection was observed in the endometrial cavity. $\mathrm{Cy}$ tologic examination of the endometrial fluid was negative for malignancy but revealed many neutrophils with scattered foamy histiocytes (Fig. 1A). Chronic active endometritis with xanthomatous inflammation was diagnosed on examination of the endometrial curettage specimen. Antibiotics were prescribed with a follow-up appointment.

Two months later, the patient presented with abdominal pain, chills, and vaginal bleeding. The patient showed signs of systemic inflammation and septic shock, with a blood neutrophil

Received: June 22, 2020 Accepted: August 18, 2020

Corresponding Author: Min Hye Kim, MD

Department of Pathology, Gyeongsang National University Hospital, 79 Gangnam-ro, Jinju 52727, Korea

Tel: +82-55-772-8061, Fax: +82-55-759-7952, E-mail: joymine86@naver.com count of $25,020 / \mathrm{mm}^{3}(95.7 \%)$ and blood pressure of $60 / 40 \mathrm{~mm}$ $\mathrm{Hg}$. Culture of an endometrial curettage specimen showed no bacterial growth, but blood culture produced Escherichia coli growth. Antibiotics were administered, and after the patient's neutrophil count and vital signs normalized, hysterectomy with right salpingo-oophorectomy was performed.

On gross examination, the endometrial cavity was filled with blood clots and most of the endometrium was bright yellow (Fig. 1B). Microscopic examination of the endometrium showed a massive aggregation of foamy histiocytes admixed with lymphocytes, neutrophils, and plasma cells (Fig. 1C). No complications related to XGE have been noted in the 4 years since surgery.

\section{Case 2}

A high-grade squamous intraepithelial lesion was detected on the cervicovaginal smear of a 72 -year-old woman. Ultrasound scanning revealed intrauterine fluid accumulation, and pyometra was suspected. Cytologic examination of the endometrial washing specimen revealed many neutrophils with scattered foamy histiocytes, and a few atypical cells, probably from the uterine cervix (Fig. 2A). Blood neutrophil count was 9,840/ $\mathrm{mm}^{3}$ (81.8\%). Endometrial fluid culture produced Pseudomonas aeruginosa growth. Hysterectomy with bilateral salpingo-oophorectomy was performed.

On gross examination, the endometrium was thickened and yellow in many areas (Fig. 2B). There was a 5.2-cm-sized fibro$\mathrm{ma}$ in the right ovary. Microscopic examination showed massive aggregation of foamy histiocytes and lymphocytes in the endometrium (Fig. 2C). Early invasive squamous carcinoma was noted in the uterine cervix. The patient has been healthy for the 23 months since surgery. 

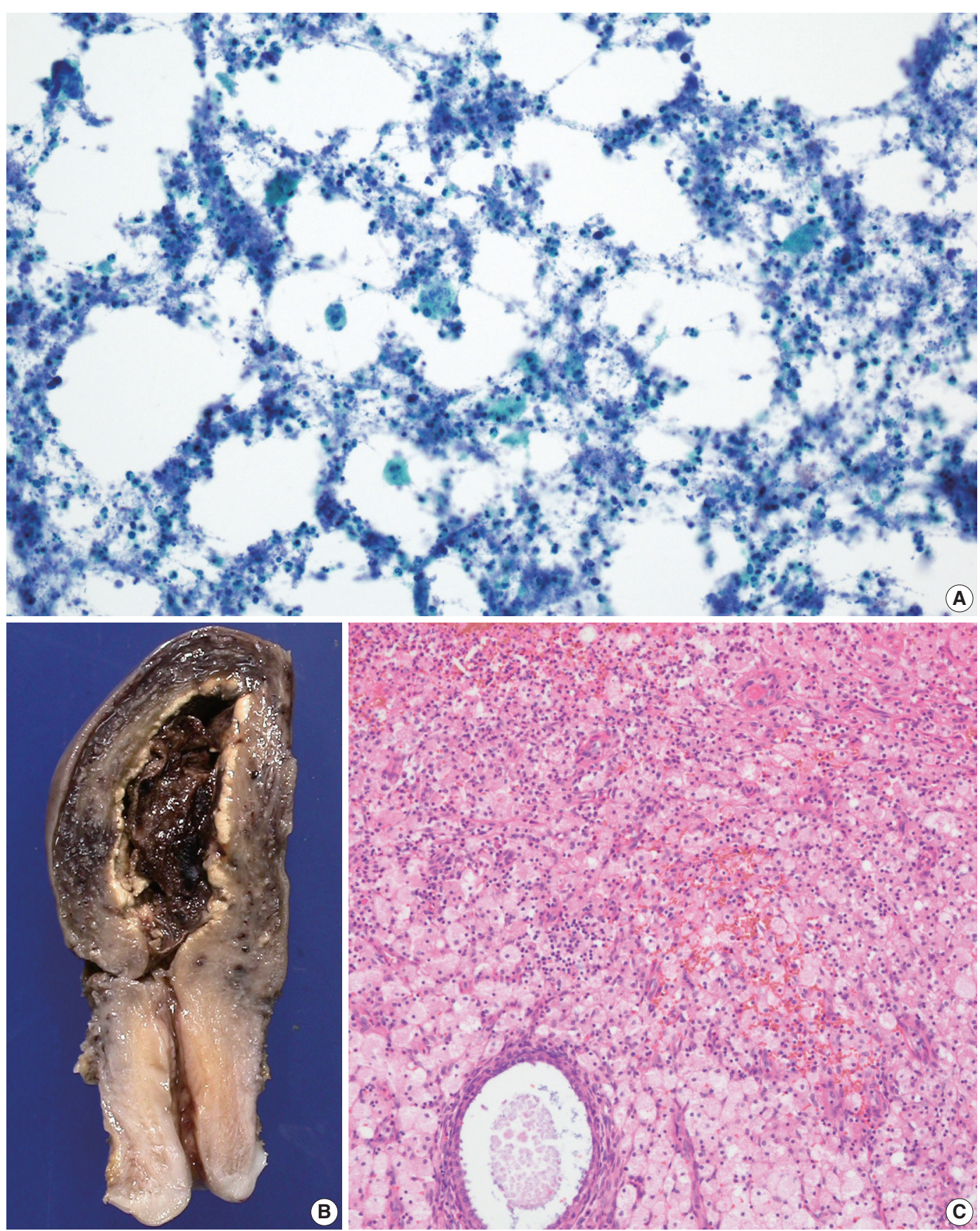

Fig. 1. (A) Aspiration cytology of endometrium showing many neutrophils with scattered foamy histiocytes. (B) Gross examination of the uterus shows bright yellow endometrium. (C) Microscopic examination of the endometrium shows aggregation of foamy histiocytes admixed with lymphocytes, neutrophils, and plasma cells.

\section{DISCUSSION}

XGE occurs mostly in postmenopausal women and is frequently associated with hematometra or pyometra. It can also occur after radiotherapy-induced endometrial or cervical tumor necrosis [1]. Cervical stenosis is presumed to contribute to the retention of hemorrhage or necrotic tissue [2].
The characteristic histological finding of XGE is the replacement of the endometrium by thick sheets of lipid-laden foamy histiocytes, although other inflammatory cells can also be present. In both of our cases, cytologic evaluation revealed many neutrophils with scattered foamy histiocytes, as seen on the microscopic findings of curettage and surgical specimens. Bacteria may or may not be isolated, but when related to bacterial infection, the 

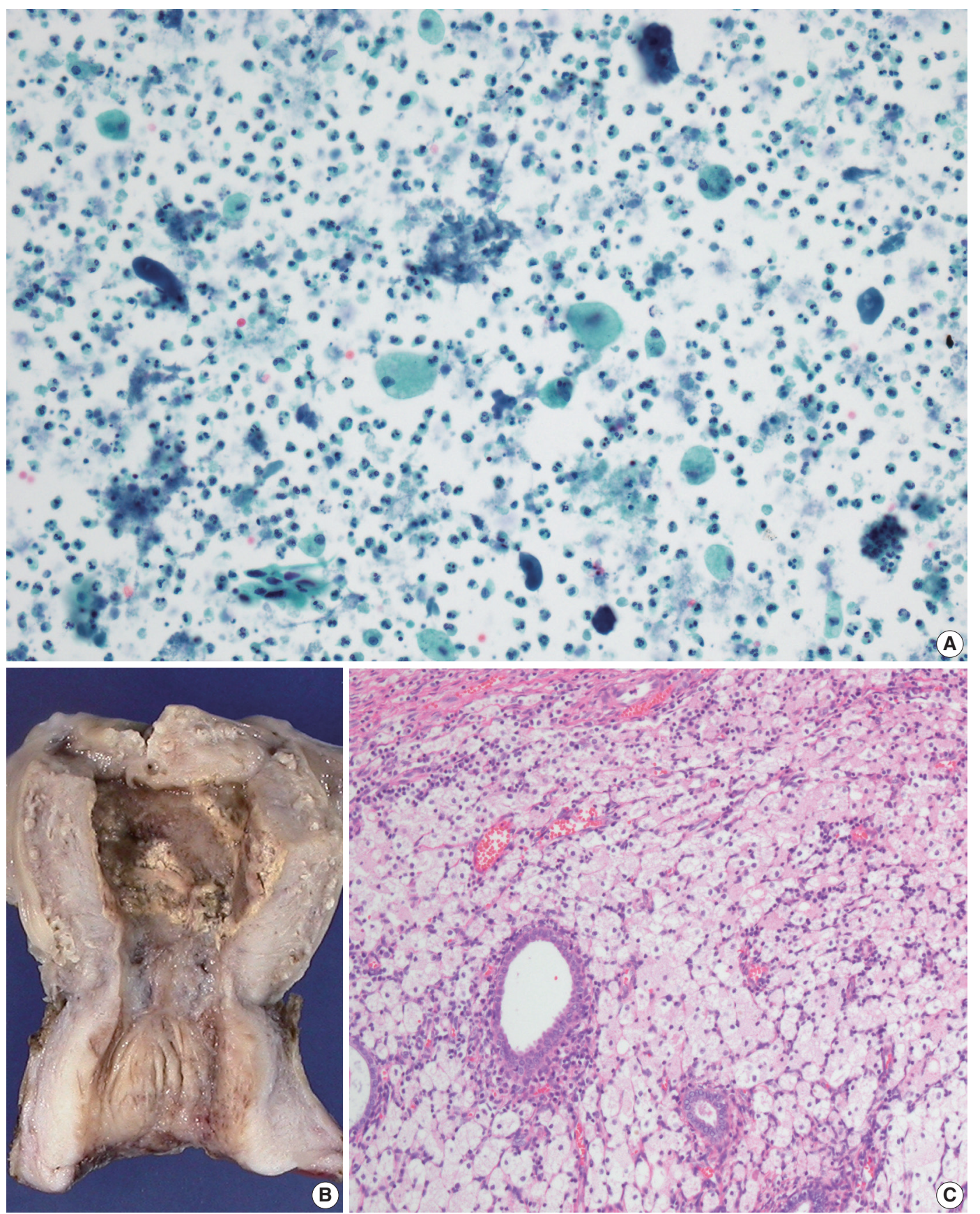

Fig. 2. (A) Endometrial washing cytology showing many neutrophils with scattered foamy histiocytes. (B) Gross examination of the uterus shows thickened and partly yellow endometrium. (C) Microscopic examination of the endometrium shows aggregation of foamy histiocytes and lymphocytes.

involved organisms include Escherichia coli, Proteus vulgaris, Peptostreptococcus magnus, or Enterococcus spp. [3,4].

While cases of XGE might resolve spontaneously or with antibiotic treatment, progression to peritonitis or sepsis can lead to a poor prognosis. Systemic inflammation originating from endometritis resulted in septic shock in our 78-year-old patient. Noack et al. [4] reported a case of XGE that led to a fatal out- come because of transmural extension of inflammation.

Differential diagnoses of XGE include malakoplakia, also a rare entity of the female reproductive tract. The absence of pathognomonic Michaelis-Gutmann bodies can exclude a diagnosis of malakoplakia [5]. On radiologic imaging, XGE can mimic malignant neoplasia of the endometrium, with findings such as endometrial thickening or mass formation [6]. However, the pres- 
ence of XGE does not exclude the possibility of co-existing malignancies [7]; therefore, extensive sectioning and thorough microscopic examination of the whole endometrium is recommended.

In conclusion, XGE is a rare but clinically important entity because of its capacity to mimic endometrial malignancy. Cytological evaluation can be useful in cases with unclear clinical presentations. XGE can be diagnosed in the absence of cells that are definite or suspicious for malignancy and in the presence of lipid-laden histiocytes and inflammatory cells such as neutrophils.

\section{Ethics Statement}

This study was approved by the Institutional Review Board of Gyeongsang National University Hospital and waived the need for informed consent (IRB No. GNUH 2020-06-001).

\section{ORCID}

Ji Min Na https://orcid.org/0000-0003-4330-6598

Min Hye Kim https://orcid.org/0000-0002-8631-5104

Gyung-Hyuck Ko https://orcid.org/0000-0002-6721-1828

Jeong Kyu Shin https://orcid.org/0000-0001-9050-0874

\section{Author Contributions}

Conceptualization: MHK, GHK. Data curation: JMN, GHK. Investigation GHK, JKS. Resources: GHK, JKS. Supervision: GHK. Visualization: JMN, GHK. Writing_original draft: JMN, GHK. Writing—review \& editing: MHK, GHK.

\section{Conflicts of Interest}

The authors declare that they have no potential conflicts of interest to disclose.

\section{References}

1. Russack V, Lammers RJ. Xanthogranulomatous endometritis: report of six cases and a proposed mechanism of development. Arch Pathol Lab Med 1990; 114: 929-32.

2. Ladefoged C, Lorentzen M. Xanthogranulomatous inflammation of the female genital tract. Histopathology 1988; 13: 541-51.

3. Barua R, Kirkland JA, Petrucco OM. Xanthogranulomatous endometritis: case report. Pathology 1978; 10: 161-4.

4. Noack F, Briese J, Stellmacher F, Hornung D, Horny HP. Lethal outcome in xanthogranulomatous endometritis. APMIS 2006; 114: 386-8.

5. Pounder DJ, Iyer PV. Xanthogranulomatous endometritis associated with endometrial carcinoma. Arch Pathol Lab Med 1985; 109: 73-5.

6. Dogan-Ekici AI, Usubutun A, Kucukali T, Ayhan A. Xanthogranulomatous endometritis: a challenging imitator of endometrial carcinoma. Infect Dis Obstet Gynecol 2007; 2007: 34763.

7. Gupta P, Dhingra KK, Roy S, Khurana N, Azad M. Xanthogranulomatous endometritis coexisting with carcinoma cervix. ANZ J Surg 2009; 79: 498-9.

8. Makkar M, Gill M, Singh D. Xanthogranulomatous endometritis: an unusual pathological entity mimicking endometrial carcinoma. Ann Med Health Sci Res 2013; 3(Suppl 1): S48-9.

9. Malik CA, Dudani S, Mani BN. Xanthogranulomatous endometritis presenting as pyometra and mimicking carcinoma on imaging. J Midlife Health 2016; 7: 88-90.

10. Du XZ, Lu M, Safneck J, Baker P, Dean E, Mottola J. Xanthogranulomatous endometritis mimicking endometrial carcinoma: A case report and review of literature. Radiol Case Rep 2019; 14: 121-5.

\section{Funding Statement}

No funding to declare. 